\title{
Zirkadiane Uhren in Gehirn und Peripherie: biologische Funktion und Relevanz für die Klinik
}

\author{
Biological role and clinical relevance of central and peripheral circadian clocks
}

\author{
Autoren \\ J. Husse ${ }^{1}$ A. Leliavski ${ }^{1}$ H. Oster ${ }^{1,2}$ \\ Institut \\ ${ }^{1}$ Max-Planck-Institut für biophysikalische Chemie Göttingen \\ 2 Medizinische Klinik I, Universität zu Lübeck
}

\section{Einleitung}

Um sich an im Tagesverlauf veränderliche Umweltbedingungen optimal anzupassen, haben die meisten Organismen interne, sog. zirkadiane Uhren (lat.: circa diem - ungefähr ein Tag) entwickelt. Fast alle physiologischen Prozesse im menschlichen Körper werden von solchen Uhren reguliert. Der vielleicht offensichtlichste zirkadiane Rhythmus ist der Schlaf-Wach-Rhythmus, aber auch viele endokrine Parameter - wie zum Beispiel die Produktion von Cortison oder Melatonin - und auch zentralnervöse Funktionen zeigen einen klaren, endogen regulierten Tagesrhythmus. Dass diese Rhythmen - auch im Menschen - endogen reguliert sind und nicht allein auf äußere Zeitgeber reagieren, konnte Jürgen Aschoff mit seinen berühmten „Bunker-Experimenten“ nachweisen, in denen er zeigte, dass viele Verhaltens- und physiologische Rhythmen auch in vollständiger Isolation und Abwesenheit von irgendwelchen Zeitsignalen weiter erhalten bleiben [1].

Werden diese Rhythmen gestört, führt das zu erheblichen gesundheitlichen Beeinträchtigungen. Den zahlreichen Symptomen, die unter dem Begriff ,Jetlag“ zusammengefasst werden, liegt eine Desynchronisation zwischen innerer Uhr und externer (Tages-)Zeit zugrunde. Auch bei Schichtarbeitern, die gezwungen werden, entgegen ihrer inneren Uhr nachts aktiv zu sein, führt die Störung der zirkadianen Rhythmik zu einer erhöhten Prävalenz von Herz-Kreislauf-Symptomen, metabolischen Erkrankungen wie Übergewicht und Typ-2-Diabetes und sogar Krebs [2]. Tierexperimentelle Studien belegen den kausalen Zusammenhang zwischen zirkadianer Störung und dem Auftreten der oben genannten Krankheiten [5]. Der Einfluss ist dabei wechselseitig: Viele Krankheiten (u.a. Krebs, Metabolisches Syndrom, Schizophrenie, Depressionen oder Morbus Alzheimer) beeinflussen das zirkadiane System, was sich zum

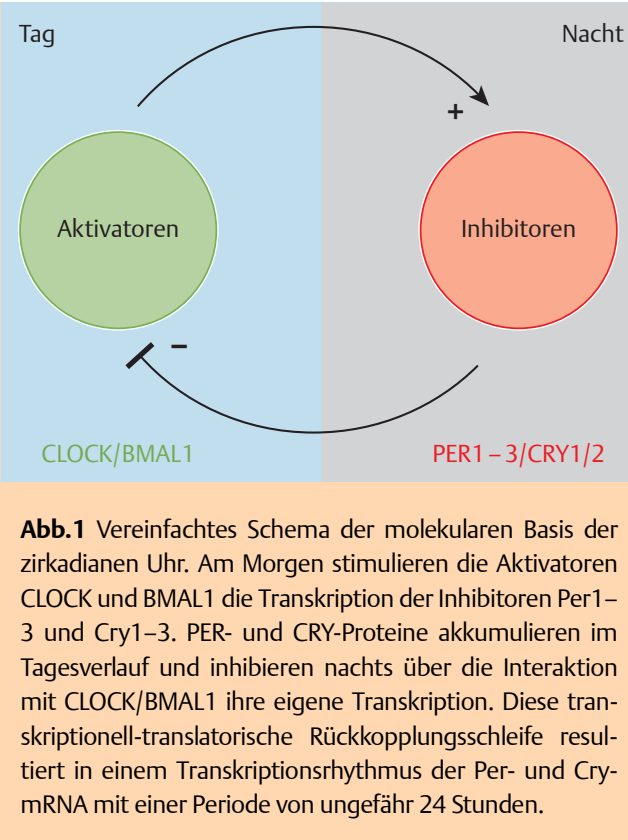

Physiologie

Schlüsselwörter

zirkadiane Rhythmen

periphere Uhren

Chronophysiologie

Chronomedizin

Keywords

circadian rhythms

peripheral clocks

$\checkmark$ chronophysiology

chronomedicine

Beispiel in Störungen des Schlaf-Wach-Rhythmus äußert [17]. Die Frage, inwiefern man den Verlauf und die Ausprägung solcher Krankheiten durch die Wiederherstellung regelmäßiger zirkadianer Rhythmen verbessern kann, ist daher von aktueller Bedeutung für Forschung und Klinik.

\section{Die molekulare Uhr $\nabla$}

Auf molekularer Ebene basieren zirkadiane Uhren auf transkriptionell-translatorischen Rückkopplungsschleifen (Abb.1). Die basic-helix-loophelix-Proteine CLOCK und BMAL1 binden an sog. E-Box-Elemente in den Promotoren der Period(Per1-3) und Cryptochrome-Gene (Cry1/2) und aktivieren so deren Transkription. Im Tagesverlauf akkumulieren PER- und CRY-Proteine im Zytoplasma, von wo aus sie nachts in den Zellkern translozieren, um dort über Interaktion mit CLOCK/BMAL1 ihre eigene Transkription zu inhi- eingereicht 27.08 .2012 akzeptiert 22.11 .2012

Bibliografie

Dol 10.1055/s-0032-1332901 Dtsch Med Wochenschr 2013; 138: 493-496 - (c) Georg Thieme Verlag KG · Stuttgart · New York - ISSN 0012-0472

Korrespondenz Prof. Dr. Henrik Oster Medizinische Klinik I, UKSH Campus Lübeck Ratzeburger Allee 160 23538 Lübeck

Tel. 0551/201-2738

Fax 0551/201-2715

eMail henrik.oster@uksh.de 


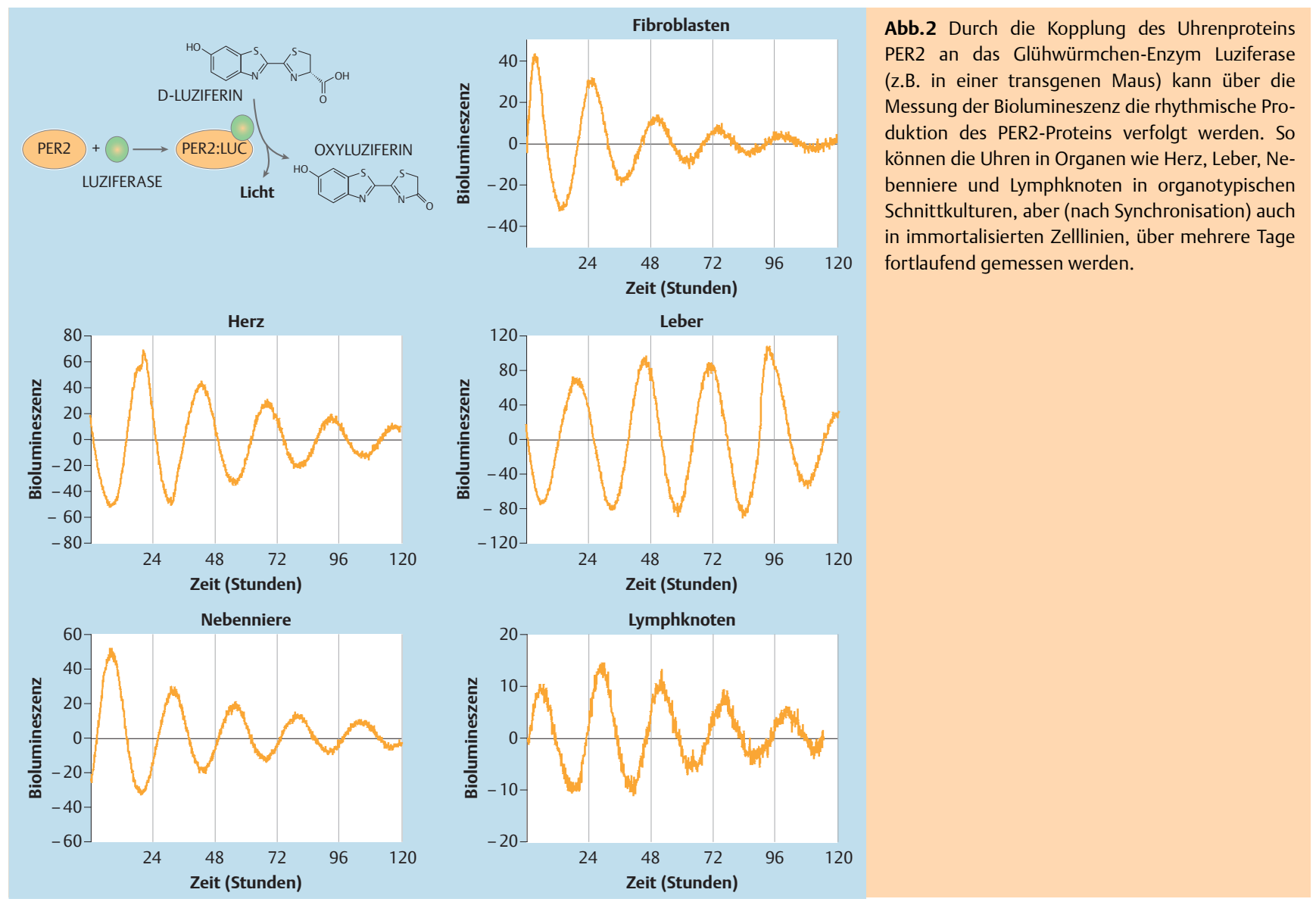

bieren. Ein allmählicher Abbau der PER/CRY-Komplexe zum Ende der Nacht hin macht CLOCK/BMAL1 frei für eine erneute Aktivierung der Per/Cry-Transkription am nächsten Morgen ( Abb.1). Daneben sind zahlreiche weitere Uhrenkomponenten bekannt, die zusätzliche Rückkopplungsschleifen bilden oder die Stabilität der Uhrenproteine regulieren und damit die Geschwindigkeit der Uhr bestimmen [11]. Durch die Messung der Expression dieser Uhren-Gene über den Tag können Phase und Amplitude der molekularen Uhr bestimmt werden. Der Chronotyp eines Menschen beschreibt die Phasenlage der inneren Uhr und wird normalerweise über die Messung der Schlaf- und Wachzeiten mittels Fragebögen bestimmt. Es werden frühe („Lerchen“) und späte Chronotypen („Eulen“) unterschieden [14].

\section{Das zirkadiane System}

Die zentrale zirkadiane Uhr des Menschen befindet sich im Nucleus suprachiasmaticus ( $\mathrm{SCN}$ ) des ventromedialen Hypothalamus. Neuere Studien konnten zeigen, dass nicht nur die Neuronen des SCN Uhren-Gene rhythmisch exprimieren, sondern dass wahrscheinlich in jeder Zelle des Körpers eine eigene zirkadiane Uhr tickt [11]. Selbst immortalisierte Fibroblastenkulturen zeigen nach Synchronisierung zirkadiane Genexpressionsrhythmen, welche über mehrere Tage hinweg anhalten, bis kleine Unterschiede in der internen Periodik der einzelnen Zellen zu einer interzellulären Desynchronisation führen. Molekulare zirkadiane Rhythmen konnten in unterschiedlichen Organen wie Leber, Niere, Nebenniere, Herz und Lunge nachgewiesen werden, z.B. durch genetische Kopplung von Luziferase an das Uhrenprotein PER2 ( Abb.2). Obwohl solche peripheren Uhren in Kultur en- dogen rhythmisch sind, werden sie im Organismus von der Zentraluhr im SCN sowohl untereinander als auch mit dem externen Tagesrhythmus synchronisiert [4].

Die Anpassung des zirkadianen Systems an die externe Zeit kann durch verschiedene Stimuli (sog. Zeitgeber) erfolgen. Der wichtigste zirkadiane Zeitgeber ist das Licht. Lichtinformationen werden vom Auge über den retino-hypothalamischen Trakt an den SCN weitergeben, der diese verarbeitet und als Zeitsignal an die Peripherie weitergibt. Periphere Uhren können zusätzlich durch Nahrung synchronisiert werden ( Abb.3). Tierexperimentelle Studien zeigen, dass eine Verschiebung der Nahrungsaufnahme in die inaktive Phase des Tages zu einer Entkoppelung zentraler und peripherer Uhren führt, was mit negativen physiologischen Konsequenzen verbunden ist [3]. So haben Nachtschichtarbeiter und Patienten mit „Night Eating Syndrome“ eine erhöhte Chance, übergewichtig zu werden oder an Diabetes zu erkranken. Die Frage, inwiefern soziale Stimuli als Zeitgeber für die zirkadiane Uhr dienen können, ist umstritten [10].

Das Uhrwerk reguliert die Zellphysiologie über die tagesrhythmische Aktivierung uhrenkontrollierter Gene. In jedem Organ sind ungefähr 10\% aller aktiv transkribierten Gene zirkadian reguliert. Interessanterweise sind diese Gene gewebsspezifisch. So kann die zirkadiane Uhr der Leber zum Beispiel über die Regulation von Genen, die im Kohlenhydratstoffwechsel involviert sind, die Glukose-Homöostase beeinflussen [6]. Ähnlich wird in der Nebenniere die Ausschüttung des Hormons Cortison durch rhythmisch regulierte Genprodukte, die in der Cortison-Biosynthese eine wichtige Rolle spielen, kontrolliert [13]. 


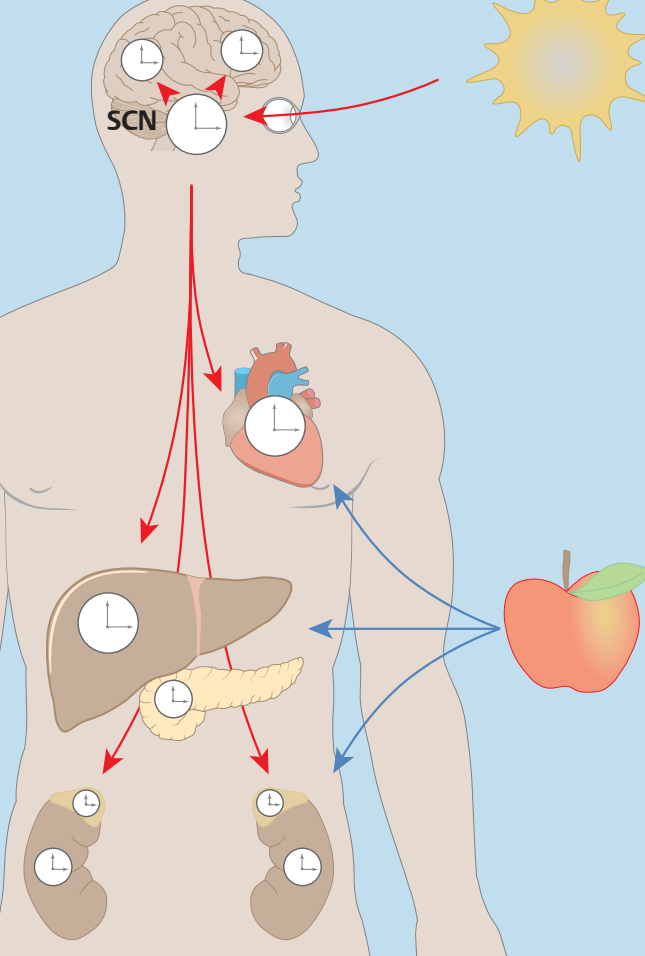

Abb.3 Das zirkadiane System des Menschen. Zirkadiane „Uhren“ ticken in so unterschiedlichen Geweben wie dem Gehirn, dem Herzen, der Leber, dem Pankreas, der Niere oder der Nebenniere. Diese Uhren müssen mit der Umwelt synchronisiert werden. Der wichtigste Zeitgeber für das zirkadiane System des Menschen ist das Licht: Es synchronisiert die Zentraluhr im Nucleus suprachiasmaticus (SCN), der dann dieses Zeitsignal an die Peripherie weitergibt. Periphere Uhren können zudem direkt über die Nahrungsaufnahme synchronisiert werden.

\section{Periphere Uhren: Endokrinum}

Welche Funktion den einzelnen peripheren Uhren in der Regulation physiologischer Funktionen zukommt, wurde in den letzten Jahren primär in tierexperimentellen Studien, z.B. an transgenen Mäusen, untersucht. So konnten wir zeigen, dass der Nebennierenuhr eine wichtige Rolle in der Regulation der tagesrhythmischen Cortisonausschüttung zukommt. Uhren-Gene im adrenergen Cortex regulieren die Sensitivität der Nebenniere für Adrenocorticotropin (ACTH) im Tagesverlauf - und damit letztendlich die Rhythmik der Cortison-Biosynthese [13]. In Abwesenheit der Nebennierenuhr ist der Tagesrhythmus der Cortisonausschüttung stark gedämpft. Ob dies zudem Konsequenzen für die akute Cortisonausschüttung im Zusammenhang mit der Stressantwort hat, wird zurzeit intensiv erforscht. Weiterhin reguliert die Nebennierenuhr die Synthese des Mineralkortikoids Aldosteron. Cry1/2-doppelmutante Mäuse neigen zu Bluthochdruck, was auf die gestörte Regulation der Aldosteron-Biosynthese zurückgeführt wurde [16]. Dies sind nur zwei gut untersuchte Beispiele für die rhythmische Regulation endokriner Parameter. Viele weitere Hormone zeigen tagesrhythmische Effekte in ihrer Regulation, darunter Melatonin, Prolaktin, Wachstumshormon, Schilddrüsenhormone, sowie eine Reihe von Adipokinen und Zytokinen.

\section{Periphere Uhren: Stoffwechsel und Detoxifikation}

Eine weitere wichtige Funktion peripherer Uhren ist die Regulation des Energiestoffwechsels. Mäuse, in denen die Leberuhr genetisch inaktiviert wurde, sind hypoglykämisch, was wahrscheinlich auf eine Deregulation von Glukosetransporter-Genen zurückzuführen ist [6]. Zudem sind viele Enzyme der Glykolyse und der Glukoneogenese uhrenreguliert. Uhren im Pankreas sind entscheidend an der Regulation der Insulinproduktion beteiligt [9]. So können Störungen im zirkadianen System (sei es genetisch oder extern, zum Beispiel durch Schichtarbeit) zu Veränderungen im Energiestoffwechsel führen und so die Entwicklung von Diabetes mellitus begünstigen. Des Weiteren reguliert die Leberuhr über die genetische Regulation von CytochromeP450-Enzymen hepatische Transformations- und Detoxifikationsprozesse und beeinflusst so Bioverfügbarkeit und Nebenwirkungen zahlreicher Medikamente [8]. Im klinischen Alltag wird oft noch zu wenig beachtet, wie sehr die Tageszeit Aufnahme, Aktivierung und Abbau eines Pharmakons bestimmt. Gerade bei der chemotherapeutischen Behandlung von Krebspatienten liegen in diesem Zusammenhang schon wichtige Studien vor [7].

\section{Zentrale Uhren: „Mood“ und Kognition \\ $\nabla$}

Das zirkadiane System hat außerdem wichtige Einflüsse auf zentralnervöse Funktionen. Uhren-Gen-Rhythmen konnten bereits in zahlreichen Bereichen des Gehirns detektiert werden, wo sie kognitive und Verhaltensprozesse beeinflussen. Die vielleicht offensichtlichste Funktion der zirkadianen Uhr ist die Regulation des Schlaf-Wach-Rhythmus. Daran sind Uhren im basalen und lateralen Hypothalamus (SCN, Nucleus preopticus ventrolateralis, orexigene Neurone des lateralen Hypothalamus) sowie im aufsteigenden Erregungssystem des Hirnstamms beteiligt. Kognitive Prozesse unterliegen u.a. der Regulation durch Uhren des zerebralen Kortex und des Hippocampus; und auch Stimmungszustände werden durch das zirkadiane System beeinflusst. So zeigen Clock-mutante Mäuse einen "manischen" Phänotyp, der durch Überexpression des Wildtyp-CLOCK-Proteins im Striatum behandelt werden kann [15]. Einer in den skandinavischen Ländern weit verbreiteten Form der Depression, die sog. Winterdepression oder „seasonal affective disorder“ (SAD), liegt eine zirkadiane Deregulation aufgrund der verkürzten Lichtperiode in den Wintermonaten zugrunde [12]. Chronotherapeutische Ansätze wie Lichttherapien und Melatoninsubstitution werden hier erfolgreich eingesetzt. Auch bei Schichtarbeitern, die unter einer chronischen Desynchronisation ihres zirkadianen Systems leiden, konnten solche Therapien erfolgreich zur Vermeidung von kognitiven Einbußen und Arbeitsunfällen eingesetzt werden.

\section{Chronophysiologie und Chronomedizin \\ $\checkmark$}

Im klinischen Alltag ist das zirkadiane System von doppelter Bedeutung: Zum einen ist eine fundamentale Kenntnis rhythmischer Prozesse im Körper essentiell für die Diagnostik. Ist ein Cortison-Plasmawert von $20 \mu \mathrm{g} / \mathrm{dl}$ am Morgen durchaus in Ordnung, weist derselbe Wert am Abend auf eine akut erhöhte Aktivierung der Hypothalamus-Hypophysen-Nebennierenachse (HPA-Achse) hin und sollte eventuell behandelt werden. Diese Parameter werden vom Chronotyp des Patienten beeinflusst. 
Entscheidend ist nämlich nicht ausschließlich, zu welcher Tageszeit die Probe gewonnen wurde, sondern vielmehr welcher internen Uhrzeit die Probennahme entspricht. Späte Chronotypen zeigen z.B. einen deutlich nach hinten verschobenen Cortisonrhythmus; viele andere physiologische Parameter reagieren ähnlich. Weiterhin sind Interaktionen mit externen Faktoren, z.B. Schichtarbeit oder unnatürliche Lichtexposition, zu berücksichtigen. Zum anderen beeinflusst die Phasenlage der inneren Uhr die Wirksamkeit einer therapeutischen Substanz im Körper. Über das rhythmisch regulierte metabolische System der Leber werden Pharmaka unterschiedlich schnell abgebaut und ausgeschieden. Dadurch verändert sich die jeweilige Halbwertzeit der Substanz im Körper. Auch Nebenwirkungen können unterschiedlich ausfallen, wie Studien an Krebspatienten eindrucksvoll zeigten.

\section{Fazit und Perspektive}

Wünschenswert wäre es, die Bestimmung von Funktion und Phasenlage innerer Uhren in die klinische Standardanamnese aufzunehmen. Sollte dies nicht möglich sein, ist es hilfreich, beim Patienten nach Schlaf- und Ernährungsverhalten in den letzten Tagen vor Einweisung zu fragen und sicherzustellen, dass nicht aufgrund von zirkadianen Rhythmusstörungen physiologische und pathologische Parameter verfälscht sein könnten. Zur Zeit werden bereits Testverfahren entwickelt, die schon anhand einer einfachen Blutprobe den Status des zirkadianen Systems erfassen können, so dass auf Basis der internen Phasenlage eine differenzierte Interpretation klinischer Parameter erfolgen und eine individuell chronomedizinisch angepasste Therapie entwickelt werden kann.

\section{Konsequenz für Klinik und Praxis}

Endogene zirkadiane Uhren im Gehirn und allen peripheren Geweben des Körpers regulieren physiologische und Verhaltensprozesse im 24-Stunden-Takt.

- Die Phasenlage des zirkadianen Systems ist ein wichtiger Parameter bei der Interpretation klinischer Daten und kann Einfluss auf die Bioverfügbarkeit und Verträglichkeit von Medikamenten haben.

Externe Rhythmusstörungen (z.B. Schichtarbeit oder Jetlag) können die innere Uhr beeinträchtigen und so die Entwicklung zahlreicher metabolischer und neuropsychiatrischer Erkrankungen begünstigen.

Danksagung: Diese Arbeit wurde durch die Max-Planck-Gesellschaft $(\mathrm{JH})$ sowie die Deutsche Forschungsgemeinschaft und die Volkswagenstiftung unterstützt (AL \& HO).

Autorenerklärung: Die Autoren erklären, dass sie keine finanziellen Verbindungen mit einer Firma haben, deren Produkt in diesem Artikel eine wichtige Rolle spielt (oder mit einer Firma, die ein Konkurrenzprodukt vertreibt).
Literatur

1 Aschoff J. Circadian Rhythms in Man. Science 1965; 148: 1427-1432

2 Boivin DB, Tremblay GM, James FO. Working on atypical schedules. Sleep Med 2007; 8: 578-589

3 Damiola F, Le Minh N, Preitner $N$ et al. Restricted feeding uncouples circadian oscillators in peripheral tissues from the central pacemaker in the suprachiasmatic nucleus. Genes Dev 2000; 14: 2950-2961

4 Dibner $C$, Schibler $U$, Albrecht $U$. The mammalian circadian timing system: organization and coordination of central and peripheral clocks. Annu Rev Physiol 2010; 72: 517-549

5 Kovac J, Husse J, Oster $H$. A time to fast, a time to feast: the crosstalk between metabolism and the circadian clock. Mol Cells 2009; 28 : 75-80

6 Lamia KA, Storch KF, Weitz CJ. Physiological significance of a peripheral tissue circadian clock. Proc Natl Acad Sci U S A 2008; 105: 15172 15177

7 Levi F, Okyar A, Dulong S et al. Circadian timing in cancer treatments. Annu Rev Pharmacol Toxicol 2010; 50: 377-421

8 Levi F, Schibler $U$. Circadian rhythms: mechanisms and therapeutic implications. Annu Rev Pharmacol Toxicol 2007; 47: 593-628

9 Marcheva B, Ramsey KM, Buhr ED et al. Disruption of the clock components CLOCK and BMAL1 leads to hypoinsulinaemia and diabetes. Nature 2010; 466: 627-631

10 Mistlberger RE, Skene DJ. Social influences on mammalian circadian rhythms: animal and human studies. Biol Rev Camb Philos Soc 2004; 79: 533-556

11 Mohawk JA, Green CB, Takahashi JS. Central and peripheral circadian clocks in mammals. Annu Rev Neurosci 2012; 35: 455-462

12 Monteleone P, Maj $M$. The circadian basis of mood disorders: recent developments and treatment implications. Eur Neuropsychopharmacol 2008; 18: 701-711

13 Oster H, Damerow S, Kiessling S et al. The circadian rhythm of glucocorticoids is regulated by a gating mechanism residing in the adrenal cortical clock. Cell Metab 2006; 4: 163-173

14 Roenneberg $T$, Kuehnle T, Juda $M$ et al. Epidemiology of the human circadian clock. Sleep Med Rev 2007; 11: 429-438

15 Roybal K, Theobold D, Graham A et al. Mania-like behavior induced by disruption of CLOCK. Proc Natl Acad Sci U S A 2007; 104: 6406-6411

16 Stanley SA, Gagner JE, Damanpour S et al. Radio-wave heating of iron oxide nanoparticles can regulate plasma glucose in mice. Science 2012; 336: 604-608

17 Wulff K, Gatti S, Wettstein JG et al. Sleep and circadian rhythm disruption in psychiatric and neurodegenerative disease. Nat Rev Neurosci 2010; 11: 589-599 